\title{
Cannibalism and Intraguild Predation Community Dynamics: Coexistence, Competitive Exclusion, and the Loss of Alternative Stable States
}

\author{
Benjamin J. Toscano, ${ }^{1, *}$ Vincent Hin, ${ }^{2}$ and Volker H. W. Rudolf ${ }^{1}$ \\ 1. BioSciences, Rice University, Houston, Texas 77005; 2. Institute for Biodiversity and Ecosystem Dynamics, University of Amsterdam, \\ NL-1090 GE Amsterdam, The Netherlands
}

Submitted April 7, 2017; Accepted June 9, 2017; Electronically published September 12, 2017

Online enhancements: appendix. Dryad data: http://dx.doi.org/10.5061/dryad.vj12j

\begin{abstract}
AвSTRACT: Predators often exert strong top-down regulation of prey, but in many systems, juvenile predators must compete with their future prey for a shared resource. In such life-history intraguild predation (LHIGP) systems, prey can therefore also regulate the recruitment and thus population dynamics of their predator via competition. Theory predicts that such stage-structured systems exhibit a wide range of dynamics, including alternative stable states. Here we show that cannibalism is an exceedingly common interaction within natural LHIGP systems that determines what coexistence states are possible. Using a modeling approach that simulates a range of ontogenetic diet shift scenarios along a productivity gradient, we demonstrate that only if the predator is competitively dominant can cannibalism promote coexistence by allowing prey to persist. If the prey is competitively dominant, cannibalism instead results in competitive exclusion of the predator and the loss of potential alternative stable states. Further, predator exclusion occurs at low cannibalistic preference relative to empirical estimates and is consistent across LHIGP systems in which the predator undergoes a complete diet shift or diet broadening over ontogeny. Given that prey is frequently competitively dominant in natural systems, our results demonstrate that even weak cannibalism can inhibit predator persistence, prompting exploration of mechanisms that reconcile theory with the common occurrence of such interactions in nature.
\end{abstract}

Keywords: density dependence, indirect interactions, mixed interactions, omnivory, ontogenetic niche shift.

\section{Introduction}

Conspecific individuals that vary in size or stage often differ in their ecological (i.e., functional) roles (Werner and Gilliam 1984). For example, in many predatory species, indi-

\footnotetext{
* Corresponding author; e-mail: benjamin.toscano@gmail.com. ORCIDs: Toscano, http://orcid.org/0000-0001-5413-8732; Hin, http://orcid .org/0000-0002-9497-1224; Rudolf, http://orcid.org/0000-0002-9214-2000.

Am. Nat. 2017. Vol. 190, pp. 000-000. (C) 2017 by The University of Chicago. 0003-0147/2017/19005-57659\$15.00. All rights reserved. DOI: $10.1086 / 693997$
}

viduals either switch resources or add resources to their diet as they develop (Werner and Gilliam 1984; Rudolf and Lafferty 2011; Rudolf et al. 2014). Predator populations can thus be simultaneously regulated by stage-specific interactions with different species. Furthermore, these shifts in interspecific interactions among stages are generally associated with concurrent shifts in intraspecific interactions (Miller and Rudolf 2011). Accordingly, structured systems exhibit a wider range of dynamics relative to unstructured systems, including alternative stable states (Miller and Rudolf 2011; de Roos and Persson 2013). In particular, which state or dynamics occur depends on the relative strength of different stage-specific interactions. Thus, any process, biogenic or anthropogenic, that shifts population size or stage distribution will also alter the relative strength of these interactions and therefore the capacity of predator-prey systems to transition between alternative stable states. Accordingly, the processes that govern predator-prey coexistence may fundamentally differ for structured versus unstructured predatorprey systems.

The dramatic ecological effects of stage-specific interactions are apparent in a widespread interaction module known as life-history intraguild predation (LHIGP; Pimm and Rice 1987; Walters and Kitchell 2001; Rudolf 2007; Abrams 2011; Hin et al. 2011; Toscano et al. 2016). LHIGP occurs when juvenile predators compete with their future prey (i.e., an intermediate consumer, hereafter "consumer") for a shared resource (fig. 1). Thus, LHIGP allows for competitive dynamics in addition to predation. Within LHIGP systems, juvenile and adult predators may feed on separate resources (i.e., a complete diet shift; fig. 1A; Hin et al. 2011; Toscano et al. 2016), or adults may continue to feed on the juvenile resource, thereby broadening their diet over ontogeny (fig. $1 B$ van de Wolfshaar et al. 2006; Rudolf 2007). Due to the opposing forces of competition and predation on different lifehistory stages, LHIGP systems are predicted to exhibit alter- 
A

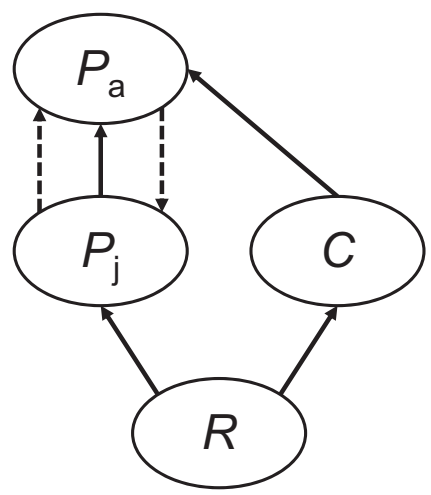

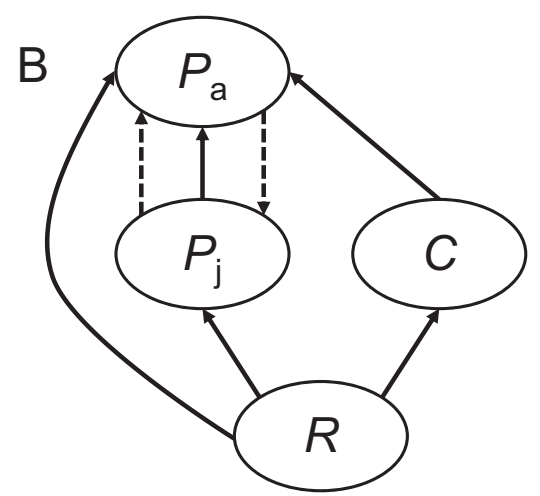

Figure 1: Life-history intraguild predation with cannibalism: complete ontogenetic diet shift $(A)$ and ontogenetic diet broadening $(B)$ scenarios. Solid arrows depict feeding relationships, while dotted arrows depict demographic transitions between predator stages (maturation and reproduction). Adult predators $\left(P_{\mathrm{a}}\right)$ feed solely on consumers $(C$; complete diet shift; $A)$ or feed equally on consumers and the resource ( $R$; diet broadening; $B$ ). In both scenarios, juvenile predators $\left(P_{\mathrm{j}}\right)$ compete with consumers for the resource and adult predators cannibalize juveniles.

native stable states: when consumers are competitively dominant, strong competition between consumers and juvenile predators can eliminate predator recruitment to the adult stage (i.e., juvenile competitive bottleneck), driving predator exclusion (a consumer-resource equilibrium; Walters and Kitchell 2001; Hin et al. 2011). Alternatively, adult predators can regulate consumers, facilitating their own recruitment and persistence (a predator-present equilibrium; van de Wolfshaar et al. 2006; Hin et al. 2011). Consequently, the long-term trajectory of LHIGP systems is potentially sensitive to predator stage structure: a preponderance of juveniles in LHIGP should free consumers from top-down control leading to predator exclusion, while a preponderance of adults and resulting topdown control should promote predator persistence.

Cannibalism (i.e., intraspecific predation) occurs in most animal taxa (Fox 1975; Polis 1981; Polis and Myers 1985), with major ramifications for both population dynamics and size/stage structure (Claessen et al. 2000, 2004; Persson et al. 2003; Rudolf 2007, 2008; Ohlberger et al. 2012). Within LHIGP systems, juvenile predators and consumers must cooccur in order to compete for a common resource, and thus adult predators frequently encounter both conspecific and heterospecific prey (Rudolf 2007; Byström et al. 2013). Furthermore, empirical studies indicate that during such encounters, LHIGP predators often preferentially consume conspecific versus heterospecific prey (fig. 2). Specifically, in a literature review of 66 experiments in which invertebrate and vertebrate predators were simultaneously offered both conspecific and heterospecific prey (fig. 2; see legend for details), only four experiments failed to measure some degree of cannibalism (i.e., Manly's $\alpha=0$ ), while 31 experiments demonstrated cannibalistic preference (i.e., Manly's $\alpha>0.5$ ). These preference estimates come from a diversity of predator taxa (crustaceans, arachnids, insects, fish, amphibians, reptiles), all of which exhibit ontogenetic diet shifts (Werner and Gilliam 1984) and thus presumably engage in LHIGP interactions in nature. The ubiquity of cannibalism and the commonness of cannibalistic preference within stage-structured predator-prey systems suggest that cannibalism must be incorporated into current LHIGP theory to better understand and predict natural LHIGP dynamics. For example, a recent study using freshwater zooplankton demonstrates a juvenile competitive bottleneck and the alleviation of this bottleneck by adult predators, the precise interactions predicted to drive alternative stable states within LHIGP systems (Toscano et al. 2016). This study further shows that cannibalism modifies the strength and qualitative outcomes of these interactions (Toscano et al. 2016). For this empirical LHIGP system (Toscano et al. 2016) and many others (fig. 2), we currently lack theory to predict how the alteration of these short-term interactions by cannibalism might scale up to influence longterm LHIGP dynamics.

Cannibalism could have strong effects on long-term LHIGP dynamics. Within LHIGP systems, predators often feed in a size-dependent manner (Pimm and Rice 1987), becoming cannibalistic at the transition from resource to prey (i.e., consumer) feeding (Rudolf 2007; Byström et al. 2013). Adult predators thus gain an additional food resource with cannibalism, while juvenile predators experience an additional source of mortality. Such stage-specific effects can have dramatic consequences for the distribution of biomass across stages (de Roos et al. 2007; de Roos and Persson 2013; Reichstein et al. 2015). Cannibalism is therefore an important but currently understudied factor that could determine the dynamics of LHIGP systems and possible coexistence states.

Here we examine the effects of cannibalism on LHIGP dynamics using models that account for the food dependence of development and reproduction (de Roos et al. 


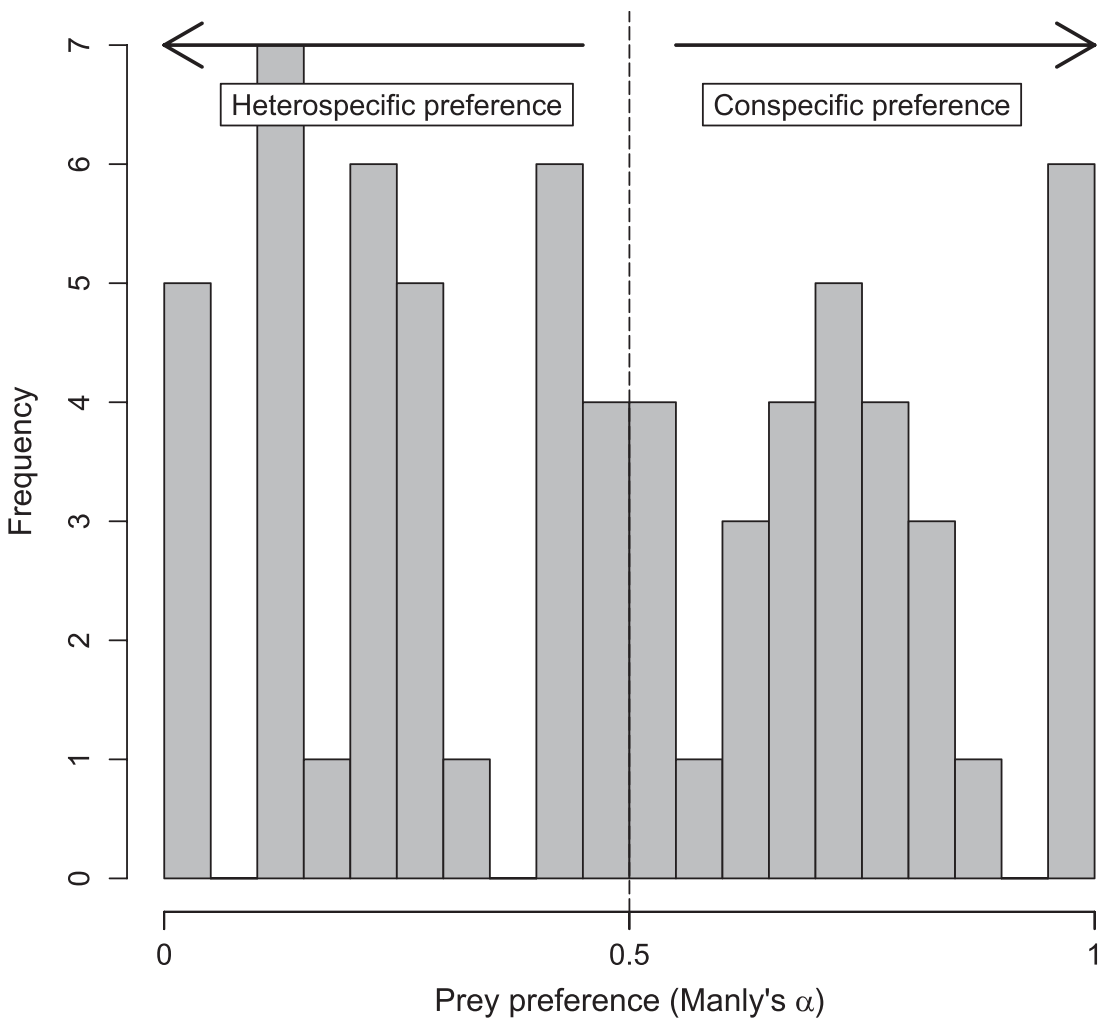

Figure 2: Cannibalistic preference estimates ( $n=66$; measured as Manly's $\alpha$; see Chesson 1978 for calculation) from empirical studies $(n=19$; see below). Manly's $\alpha$ is a measure of the probability that a conspecific or heterospecific prey item is selected when both prey types are offered simultaneously. Manly's $\alpha$ scales from 0 to 1 , with $\alpha=0.5$ indicating no preference (vertical dashed line) and $\alpha<0.5$ and $\alpha>0.5$ indicating cannibalistic aversion and preference, respectively. When Manly's $\alpha$ or data necessary to calculate Manly's $\alpha$ were not reported directly, a plot digitizer (Web Plot Digitizer 3.8) was used to extract relevant data from graphs. Empirical studies were found with a literature search conducted in March 2017 on Google Scholar. Search terms were "cannibalistic" or "cannibalism" combined with "preference," "choice," "heterospecific," "interspecific," "Manly's," and "Chesson." Empirical studies: Duelli (1981); Orr et al. (1990); Leonardsson (1991); Streams (1992); Macpherson and Gordoa (1994); MacRae and Croft (1997); Baras et al. (1999); Schausberger (1999); Walzer and Schausberger (1999); Gerber and Echternacht (2000); Schausberger and Croft (2000); Yasuda et al. (2001); Gagné et al. (2002); Santi et al. (2003); Park et al. (2005); Dörner et al. (2007); Mahe et al. (2007); Rudolf (2008); Byström et al. (2013).

2007; de Roos and Persson 2013). We explore the effects of cannibalism on LHIGP dynamics when adult predators exhibit either (1) a complete diet shift (juveniles and adults feed on separate food resources; fig. $1 \mathrm{~A}$ ) or (2) diet broadening over ontogeny (adults continue to feed on the resource of juveniles; fig. $1 B$ ). Both patterns of ontogenetic diet change are common in nature (Werner and Gilliam 1984; Werner 1988; Rudolf and Lafferty 2011), allowing us to assess the generality of potential effects.

We model cannibalism as a single parameter that represents the preference for conspecific versus heterospecific prey. Cannibalistic preference has been estimated empirically for a diversity of predator species (fig. 2), allowing us to make general predictions for how these cannibalistic predators might influence the long-term dynamics of the LHIGP systems in which they occur. We found that even weak cannibalism can inhibit predator persistence in both LHIGP sys- tem variants through a reduction in the total biomass of adult predators and thus the top-down control of competitively dominant consumers. We further discuss mechanisms that may facilitate predator persistence in LHIGP systems with cannibalism, thus reconciling our findings with the apparently common occurrence of such interactions in nature.

\section{Model and Methods}

\section{Model Formulation}

We examine the effects of cannibalism on LHIGP dynamics by using a stage-structured, bioenergetics modeling approach (see also Yodzis and Innes 1992; de Roos et al. 2007). Using this approach, Hin et al. (2011) analyze a model of LHIGP in which the predator undergoes a complete ontogenetic diet shift. We build upon this model to explore the effects of can- 
nibalism on both complete diet shift and diet broadening LHIGP scenarios. Two ordinary differential equations (ODEs) keep track of juvenile $\left(P_{\mathrm{j}}\right)$ and adult predator $\left(P_{\mathrm{a}}\right)$ total biomass densities (mass per unit volume; table 1). Despite a simple two-stage population structure, this formulation, under equilibrium conditions, yields predictions identical to that of a physiologically structured population model that explicitly accounts for a continuous population size distribution and mass-specific individual-level physiological rates (de Roos et al. 2007, 2008).

Total biomass densities of unstructured consumer and resource populations, denoted $C$ and $R$, respectively, are modeled with separate ODEs (table 1). In the absence of the consumer and the predator, the resource exhibits semichemostat growth $\delta\left(R_{\max }-R\right)$, with $\delta$ and $R_{\max }$ the turnover rate and maximum resource biomass, respectively (table 1 ). Resource productivity, $\delta R_{\max }$, increases linearly with $R_{\max }$, and so we use $R_{\max }$ as a proxy for productivity throughout the model analysis (see "Model Parameterization and Analysis"). Consumers, juvenile predators, and adult predators reduce total resource biomass through feeding (table 1).

Resource consumption by consumers increases their massspecific net biomass production,

$$
v_{c}(R)=\sigma m_{\mathrm{c}} \frac{R}{h+R}-t_{\mathrm{c}},
$$

where $\sigma$ represents the conversion efficiency of resources consumed with a type II functional response $\left(m_{\mathrm{c}}\right.$ and $h$ represent the mass-specific maximum consumption rate of the consumer and the half-saturation constant, respectively) balanced by the mass-specific maintenance rate of the consumer, $t_{c}$. Thus, basic metabolic demands are met before energy is allocated to reproduction. Conversion efficiency, $\sigma$, represents the net effects of assimilation efficiency (i.e., ingestion lost to feces and urine) and specific dynamic action (i.e., thermal energy expenditure due to food processing; Peters 1983). Consumer biomass is reduced by adult predators (juvenile predators do not feed on the consumer in either LHIGP scenario; fig. 1) and background mortality, $\mu_{\mathrm{c}}$ (table 1).

Juvenile and adult predator stages are directly linked by maturation, reproduction, and asymmetric cannibalism (juveniles cannot consume adults; fig. 1). The mass-specific net biomass production of juvenile predators,

$$
v_{\mathrm{j}}(R)=\sigma m_{\mathrm{p}} \frac{R}{h+R}-t_{\mathrm{p}},
$$

also increases with resource consumption with a type II functional response $\left(m_{\mathrm{p}}\right.$ represents the mass-specific maximum consumption rate of the predator) balanced by the massspecific maintenance rate of the predator, $t_{\mathrm{p}}$. Juvenile predator biomass is reduced by the juvenile mortality rate $(\omega$, defined in eq. [5]) and altered by demographic transitions between juvenile and adult predator stages (table 1) as follows.

The net biomass production of juveniles, $v_{\mathrm{j}}(R)$, is spent on juvenile growth, which adds biomass to the juvenile stage. Conditional upon survival, growth ultimately leads to maturation and transfer of juvenile biomass to the adult stage. The mass-specific maturation rate equals

$$
\gamma\left(v_{\mathrm{j}}(R)\right)=\frac{v_{\mathrm{j}}(R)-\omega}{1-z^{1-\omega / v_{\mathrm{j}}(R)}}
$$

and ensures a correct translation between the biomass densities of juvenile and adult predators and a continuously sizestructured model under equilibrium conditions from which this stage-structured model is derived (de Roos et al. 2008). Mass-specific maturation increases with net juvenile biomass production and decreases with $z$, the ratio between predator size at birth and that at maturation, as well as the juvenile mortality rate, $\omega$. Maturation represents the sole biomass input into the adult predator equation, as adults spend all net production on reproduction and are assumed not to grow. Total adult biomass is reduced by predator background mortality $\left(\mu_{\mathrm{p}}\right.$; table 1$)$.

Table 1: Model equations for life-history intraguild predation with asymmetric cannibalism (adults consume juveniles)

\begin{tabular}{ll}
\hline Description & \multicolumn{1}{c}{ Dynamic equation } \\
\hline Resource biomass & $\frac{d R}{d t}=\delta\left(R_{\max }-R\right)-m_{\mathrm{c}} \frac{R}{h+R} C-m_{\mathrm{p}} \frac{R}{h+R} P_{\mathrm{j}}-m_{\mathrm{p}} \frac{(1-\Phi) R}{h+(1-\Phi) R+\Phi\left(C+\beta P_{\mathrm{j}}\right)} P_{\mathrm{a}}$ \\
Consumer biomass & $\frac{d C}{d t}=v_{\mathrm{c}}(R) C-m_{\mathrm{p}} \frac{\Phi C}{h+(1-\Phi) R+\Phi\left(C+\beta P_{\mathrm{j}}\right)} P_{\mathrm{a}}-\mu_{\mathrm{c}} C$ \\
Juvenile predator biomass & $\frac{d P_{\mathrm{j}}}{d t}=v_{\mathrm{a}}\left(R, C, P_{\mathrm{j}}\right) P_{\mathrm{a}}+v_{\mathrm{j}}(R) P_{\mathrm{j}}-\gamma\left(v_{\mathrm{j}}(R)\right) P_{\mathrm{j}}-\omega P_{\mathrm{j}}$ \\
Adult predator biomass & $\frac{d P_{\mathrm{a}}}{d t}=\gamma\left(v_{\mathrm{j}}(R)\right) P_{\mathrm{j}}-\mu_{\mathrm{p}} P_{\mathrm{a}}$ \\
\hline
\end{tabular}

Note: Submodels are presented in the main text. $\delta=$ resource turnover rate; $R_{\max }=$ maximum resource biomass $m_{\mathrm{c}}=$ consumer maximum consumption rate; $m_{\mathrm{p}}=$ predator maximum consumption rate; $h=$ half-saturation constant; $\Phi=$ adult predator diet; $\beta=$ cannibalistic preference; $v_{c}(R)=$ consumer net biomass production; $\mu_{\mathrm{c}}=$ consumer background mortality; $v_{\mathrm{a}}\left(R, C, P_{\mathrm{j}}\right)=$ adult predator net biomass production; $v_{\mathrm{j}}(R)=$ juvenile predator net biomass production; $\gamma\left(v_{j}(R)\right)=$ predator maturation rate; $\omega=$ juvenile predator mortality rate; $\mu_{\mathrm{p}}=$ predator background mortality. 
The net biomass production of adults,

$$
v_{\mathrm{a}}\left(R, C, P_{\mathrm{j}}\right)=\sigma m_{\mathrm{p}} \frac{(1-\Phi) R+\Phi\left(C+\beta P_{\mathrm{j}}\right)}{h+(1-\Phi) R+\Phi\left(C+\beta P_{\mathrm{j}}\right)}-t_{\mathrm{p}},
$$

increases with consumption of resource, consumer, and juvenile predator biomass according to a type II functional response. The parameter $\Phi$ represents the fraction of time the adult predator spends foraging on the resource versus conspecific and heterospecific (i.e., consumer) prey: at $\Phi=1$, the adult predator forages solely on prey (i.e., complete diet shift, with cannibalism depending on $\beta$; fig. $1 A$ ), while at $\Phi=0.5$, the adult predator spends equal amounts of time foraging on the resource and prey (i.e., diet broadening, with cannibalism depending on $\beta$; fig. $1 B$ ). The parameter $\beta$ represents the cannibalistic preference of adult predators feeding on juveniles. This parameter scales the preference for conspecific prey relative to heterospecific prey: at $\beta=1$, adults have no preference for conspecific versus heterospecific prey, while $\beta<1$ and $\beta>1$ indicate cannibalistic aversion (i.e., heterospecific preference) and cannibalistic preference, respectively. In empirical studies, cannibalistic preference is often measured by Manly's $\alpha$ (Chesson 1978), which scales from 0 to 1 , with $\alpha=0.5$ indicating no cannibalistic preference (as presented in fig. 2; data available in the Dryad Digital Repository: http://dx.doi.org/10.5061/dryad.vj12j; Toscano et al. 2017). Thus, our $\beta=1$ corresponds to Manly's $\alpha=0.5$.

Finally, cannibalism increases the juvenile mortality rate,

$$
\omega=\Phi \beta m_{\mathrm{p}} \frac{P_{\mathrm{a}}}{h+(1-\Phi) R+\Phi\left(C+\beta P_{\mathrm{j}}\right)}+\mu_{\mathrm{p}}
$$

which sums the effects of cannibalism and background predator mortality. The juvenile mortality rate reduces juvenile biomass, $P_{\mathrm{j}}$ (table 1 ), as well as the mass-specific maturation rate, $\gamma\left(v_{j}(R)\right)$ (eq. [3]), since it determines juvenile survival and hence the fraction of newborn biomass that survives to the adult stage.

\section{Model Parameterization and Analysis}

This model was parameterized following Hin et al. (2011), which we refer the reader to for more thorough biological justification of parameter settings. Briefly, this parameterization (see fig. 3 legend for settings) assumes quarter-power scaling relationships of mass-specific parameters (Peters 1983; Yodzis and Innes 1992; Brose et al. 2006), where adult predators are assumed to be 100 times the mass of consumers. Notably, the predator maximum consumption rate, $m_{\mathrm{p}}$, is set at 2.5, while the consumer maximum consumption rate, $m_{c}$, is set at 10 . This parameterization ensures that the consumer is a superior resource competitor in both complete diet shift and diet broadening LHIGP scenarios (Hin et al. 2011). Consumer competitive superiority is likely in nature as a result of morphological or behavioral trade-offs associated with the predator ontogenetic diet shift (Toscano et al. 2016; see "Discussion" for explanation), but we later relax this condition and assume the opposite to examine the effects of cannibalism on both LHIGP scenarios when the predator is the superior resource competitor.

We used MATCONT (Dhooge et al. 2003), a MATLAB package for numerical bifurcation analysis of ODEs, to calculate equilibrium biomass densities and assess equilibrium stability (model implementation available in the Dryad Digital Repository: http://dx.doi.org/10.5061/dryad.vj12j; Toscano et al.2017). We analyze the model scenarios $\Phi=1$ and $\Phi=$ 0.5 , representing a complete ontogenetic diet shift and ontogenetic diet broadening, respectively (fig. 1). While predators in nature likely occur along a continuum between these two diets, our initial model explorations demonstrated a continuous transition between the dynamics of these different diet scenarios (not shown here). Therefore, modeling the two separate scenarios (complete diet shift and diet broadening) as we do here captures all possible qualitative dynamics. To examine potential effects of enrichment, we first explore equilibrium dynamics of these two parameterizations over a gradient in resource productivity (i.e., $R_{\max }$ ). This analysis also allows us to compare dynamics to those of classic (i.e., unstructured) tritrophic systems evaluated over gradients in productivity (Oksanen et al. 1981). We then apply numeric continuation methods to these equilibria, evaluated at $R_{\max }=$ 3 , as a function of cannibalistic preference, $\beta$, to assess how resource, consumer, and predator equilibrium biomasses respond to increasing cannibalism.

We additionally examined equilibrium states over gradients in $R_{\max }$ and $\beta$ in four different LHIGP situations (i.e., parameterizations): (1) complete diet shift with the consumer as the superior resource competitor $\left(m_{\mathrm{p}}=2.5\right)$, (2) complete diet shift with the predator as the superior resource competitor $\left(m_{\mathrm{p}}=5\right)$, (3) diet broadening with the consumer as the superior resource competitor $\left(m_{\mathrm{p}}=2.5\right)$, and (4) diet broadening with the predator as the superior resource competitor $\left(m_{\mathrm{p}}=5\right)$. These analyses allowed us to explore the effects of cannibalism across a broader spectrum of potential LHIGP systems, notably when consumers are competitively inferior to predators (in contrast to the prior analyses), and extended productivity and cannibalism gradients within each of these four systems.

\section{Results}

\section{Complete Diet Shift LHIGP Scenario}

The dynamics of LHIGP in which the predator undergoes a complete diet shift between stages (without cannibalism) 


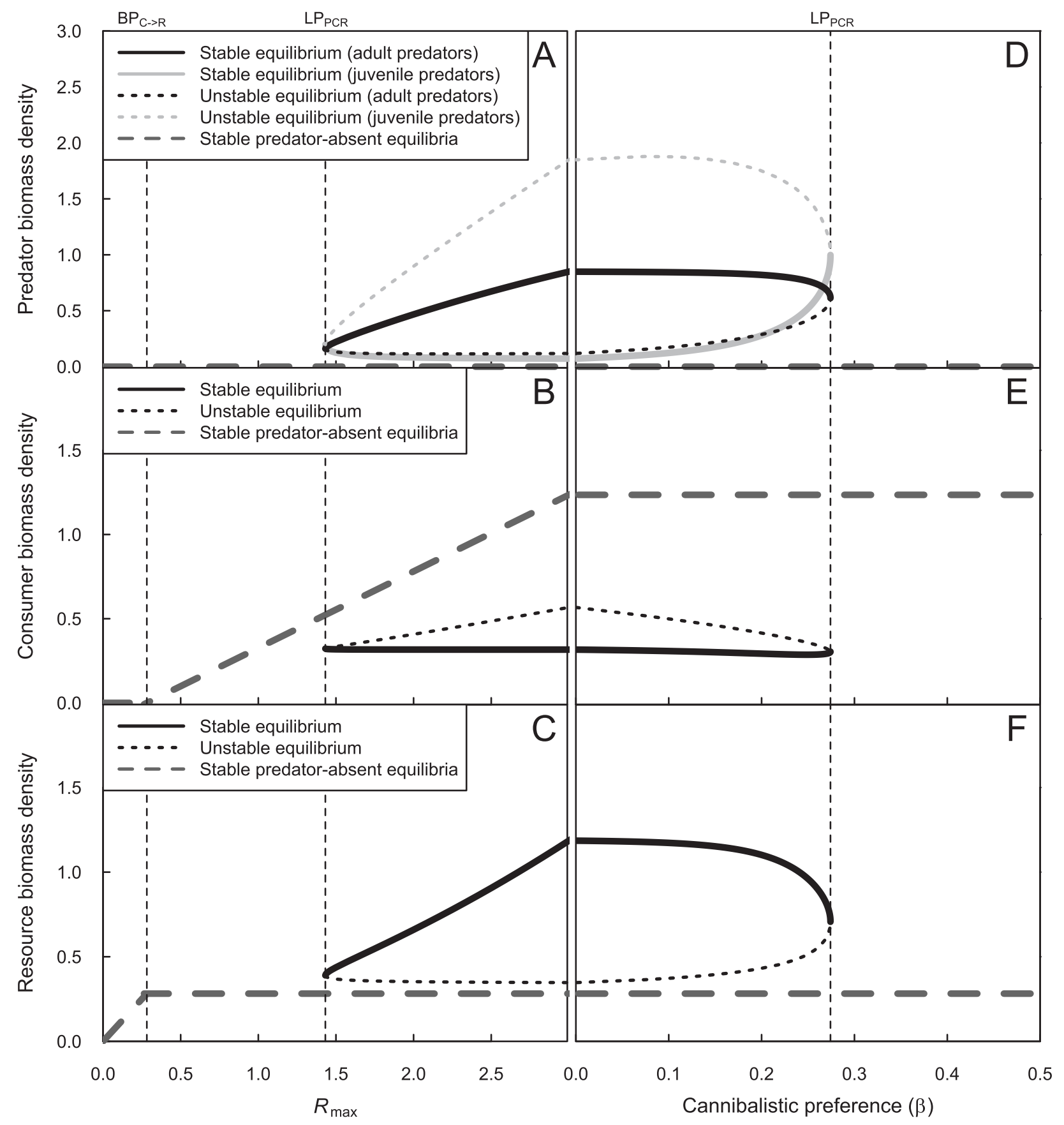

Figure 3: Equilibrium biomasses as a function of resource productivity $\left(R_{\max }\right)$ when the predator undergoes a complete ontogenetic diet shift $(A-C)$ and effects of cannibalistic preference $(\beta)$ on these equilibria $\left(D-F\right.$; calculated at $\left.R_{\max }=3\right)$. The consumer is competitively dominant in resource consumption. Juvenile predator biomass is indicated with light gray lines and adult predator biomass with black lines. Solid lines depict stable predator-present equilibria and dotted lines unstable predator-present equilibria. Dashed lines depict equilibria with zero predator density (i.e., stable resource-only and consumer-resource equilibria). Vertical (dotted) lines mark different productivity $(A-C)$ and cannibalistic $(D-F)$ thresholds at which a qualitative change in equilibria occurs. In $A-C$, the consumer invasion (branching) point, $\mathrm{BP}_{\mathrm{C} \rightarrow \mathrm{R}}$, marks the productivity threshold at which consumers invade the resource-only equilibrium, while $\mathrm{LP}_{\mathrm{PCR}}$ (a limit point) marks the lowest productivity at which coexistence is possible. In $D-F, \mathrm{LP}_{\mathrm{PCR}}$ marks the level of cannibalism at which coexistence is no longer possible. Parameter values are as follows: in $A-C, R_{\max }$ is varied, $\delta=1, \sigma=0.5, m_{\mathrm{c}}=10, m_{\mathrm{p}}=2.5, t_{\mathrm{c}}=1, t_{\mathrm{p}}=0.3, \mu_{\mathrm{c}}=0.1, \mu_{\mathrm{p}}=0.03, h=1 ; \Phi=1, z=$ $0.01, \beta=0$; in $D-F$, all parameters are the same as in $A-C$, except $R_{\max }=3$ and $\beta$ is varied. A color version of this figure is available online. 
were previously described by Hin et al. (2011). We recapitulate the dynamics here to set the stage for introducing cannibalism to this LHIGP scenario (fig. $1 A$ ).

Starting from low resource productivity $\left(R_{\max }\right)$, consumers invade the resource-only equilibrium before predators (at productivity threshold $\mathrm{BP}_{\mathrm{C} \rightarrow \mathrm{R}}$ ) because of their superior competitive ability (fig. $3 A-3 C$ ). This invasion point marks the start of a consumer-resource equilibrium that is stable (i.e., robust to predator invasion) over increasing resource productivity. At higher resource productivity, predator existence becomes possible (at productivity threshold $\mathrm{LP}_{\mathrm{PCR}}$ ) as an alternative stable predator-consumer-resource state (fig. $3 A-3 C$ ). In this coexistence state, adult predators regulate consumer biomass (fig. $3 B$ ), while resource biomass increases with $R_{\max }$ (fig. $3 C$ ). Thus, coexistence equilibrium dynamics follow those of a traditional top-down food chain (Oksanen et al. 1981; Hin et al. 2011).

The predator stage distribution is dominated by adults in this coexistence state: adult biomass increases with resource productivity, while juvenile biomass remains roughly constant (fig. 3A). This stage-specific response to resource productivity arises because juveniles feed at a higher rate than adults, and thus maturation (i.e., biomass transition to the adult stage) occurs at a faster rate than reproduction. This asymmetry forms a reproductive bottleneck behind which adult biomass accumulates (i.e., reproduction regulation), leading to an adult-dominated population structure. Specifically, juveniles feed at a higher rate than adults because their food source is more abundant than that of adults: resource biomass increases with resource productivity (fig. 3C) because adults regulate competitively dominant consumers (consumer biomass remains constant with increasing resource productivity: fig. $3 B$ ). Thus, when predators exhibit a complete diet shift in LHIGP systems, reproduction is the limiting life-history process, and the preponderance of adults (and resulting top-down control) is necessary to maintain the coexistence state.

\section{Complete Diet Shift with Cannibalism}

The consumer-resource equilibrium remains unchanged by cannibalism $(\beta)$ because of the absence of predators (fig. 3D$3 F$ ). In contrast, the three-species (predator-consumerresource) coexistence equilibrium is highly sensitive to cannibalism $(\beta)$; when a threshold of cannibalistic preference $\left(\mathrm{LP}_{\mathrm{PCR}}\right)$ is exceeded, this equilibrium is no longer possible (fig. 3D-3F). Given our model parameterization, this threshold occurs at a cannibalism level of $\beta \sim 0.27$ (i.e., heterospecific preference). At values of $\beta$ beyond $\mathrm{LP}_{\mathrm{PCR}}$, the only equilibrium possible is the consumer-resource equilibrium (fig. 3D-3F). Thus, moderate levels of cannibalism in the predator can actually prevent predator persistence in the complete diet shift LHIGP scenario, precluding potential alternative stable states.

A shift in the predator stage distribution is the mechanism behind the collapse of the coexistence state: cannibalism reduces the total biomass of adult predators but increases the total biomass of juvenile predators (fig. 3D). This seemingly counterintuitive effect, a reversal in the predator stage distribution, is potentially driven by two processes. First, cannibalism adds an exclusive food resource for adults (conspecific prey) that is not available to juveniles. Thus, cannibalism can help free adults from resource limitation, increasing the rate of reproduction relative to maturation. Second, cannibalism induces stage-specific mortality of juvenile predators. Such mortality can alter population stage distribution independent of which life stage is subjected to increased mortality (de Roos et al. 2007). As explained above, the coexistence equilibrium of predators and intermediate consumers is dependent on adult predator-driven top-down control. Accordingly, the reduction in adult predators and increase in competitively inferior juvenile predators due to cannibalism (i.e., a shift from reproduction regulation to maturation regulation; de Roos et al. 2007) reduce topdown control, leading to competitive exclusion of the predator and loss of the three-species coexistence state.

Additional analyses indicate that juvenile-specific mortality, rather than energy gain for adults, is the primary driver of the effect of cannibalism on predator stage distribution (figs. $\mathrm{A} 1 A-\mathrm{A} 1 C, \mathrm{~A} 2 A-\mathrm{A} 2 C$; figs. $\mathrm{A} 1-\mathrm{A} 3$ are available online). Even when energy gain for adult predators from cannibalism is prevented, cannibalism has the same qualitative effect on predator stage distribution (a shift from adult to juvenile biomass dominance; fig. A $1 A-\mathrm{A} 1 C$ ). Furthermore, an increase in juvenile-specific mortality alone, in the absence of cannibalism, captures this same qualitative effect (fig. A2A-A2C).Thus, it appears that juvenile-specific mortality due to cannibalism acts to shift biomass dominance to the juvenile stage, ultimately eliminating the coexistence equilibrium.

\section{Diet Broadening LHIGP Scenario}

When predators broaden their diet over ontogeny (i.e., consume both the intermediate consumer and the resource as adults; fig. $1 B$ ) alternative stable states are again possible, but these states are different from those in the complete diet shift scenario (fig. 1A). In diet broadening, consumerresource and predator-resource states co-occur over a range of $R_{\max }$. Thus, coexistence in the diet broadening scenario when the consumer is competitively dominant is not possible.

Starting at low resource productivity, competitively superior consumers again invade the resource-only equilibrium before predators (at productivity threshold $\mathrm{BP}_{\mathrm{C} \rightarrow \mathrm{R}}$ ), leading 
to a stable consumer-resource equilibrium over increasing resource productivity (fig. 4A-4C). At higher resource productivity, however, predators invade (at productivity threshold $\mathrm{BP}_{\mathrm{P} \rightarrow \mathrm{R}}$ ), and then stable predator existence (at productivity threshold $\left.\mathrm{BP}_{\mathrm{C} \rightarrow \mathrm{PR}}\right)$ becomes possible as an alternative predatorresource state (fig. $4 A-4 C$ ). While the predator-resource equilibrium branch originates at $\mathrm{BP}_{\mathrm{P} \rightarrow \mathrm{R}}$, it becomes stable only at $\mathrm{BP}_{\mathrm{C} \rightarrow \mathrm{PR}}$ as a result of an unstable coexistence equilibrium originating at this threshold (fig. 4A-4C). In this alternative stable state, adult and juvenile predators persist solely off resource biomass, regulating resource biomass with increasing resource productivity (fig. $4 C$ ).

As in the complete diet shift scenario, this predator-present state is dominated by adult biomass. Specifically, adult biomass increases at a faster rate than juvenile biomass with increasing resource productivity (fig. $4 A$ ). This occurs because generalist adults, which split their time foraging for resources and prey $(\Phi=0.5)$, feed at half the rate of juveniles in the absence of consumers. Accordingly, reproduction occurs at a lower rate than maturation, driving biomass accumulation in the adult stage. Thus, just as in the complete diet shift scenario, the predator-present state in the diet broadening scenario is reproductively regulated (dominated by adult biomass), and this condition is necessary to ward off consumer invasion.

\section{Diet Broadening with Cannibalism}

As in the complete diet shift LHIGP scenario, cannibalism $(\beta)$ has no effect on the consumer-resource equilibrium but quickly destabilizes (at the threshold in cannibalistic preference $\mathrm{BP}_{\mathrm{C} \rightarrow \mathrm{PR}}: \beta \sim 0.26$ ) the predator-present (predatorresource) state (fig. $4 D-4 F$ ). Thus, at values of $\beta$ beyond $\mathrm{BP}_{\mathrm{C} \rightarrow \mathrm{PR}}$, the only stable state possible is the consumerresource equilibrium (fig. $4 D-4 F$ ). Therefore, just as in the complete diet shift LHIGP scenario, low levels of cannibalism (i.e., maintaining a heterospecific prey preference) in the diet broadening scenario again prevent predator persistence and thus potential alternative stable states.

The mechanism behind this effect of cannibalism on community structure also involves a shift in the predator stage distribution. Specifically, where adult biomass outweighs juvenile biomass in the absence of cannibalism, cannibalism reverses this asymmetry (fig. $4 D$ ). We show that juvenilespecific mortality, and not addition of an exclusive resource for adults, is again the primary driver of the effect of cannibalism on predator stage distribution (figs. A1D-A $1 F, \mathrm{~A} 2 D-$ $\mathrm{A} 2 F)$. Thus, juvenile-specific mortality due to cannibalism releases adults from resource limitation and shifts biomass dominance to the juvenile stage. Ultimately, this reduction in adult predators and the preponderance of competitively inferior juveniles destabilize the predator-resource equilibrium (fig. 4D-4F) because predators can no longer resist consumer invasion.

\section{Importance of Consumer Competitive Ability}

The aforementioned dynamics occur when consumers are competitively dominant over predators in resource competition. We further show that these same dynamics remain qualitatively unchanged over a wider range of cannibalism $(\beta)$ and resource productivity $\left(R_{\max }\right)$ values (fig. $\left.5 A, 5 B\right)$. Specifically, predator persistence is limited to low cannibalistic preference and higher resource productivity in both complete diet shift (fig. $5 A$ ) and diet broadening (fig. 5B) LHIGP scenarios. At high cannibalistic preference and low resource productivity, a consumer-resource state is the only possible equilibrium (fig. $5 A, 5 B$ ), because of competitive exclusion of predators.

In contrast, when the predator is superior in resource competition, a juvenile competitive bottleneck - and thus predator exclusion - is no longer possible. Instead, a predator-present equilibrium always occurs at low resource productivity and remains robust to cannibalism (fig. $5 C, 5 D$ ). Specifically, in the complete diet shift scenario, the consumer invades the resource-only equilibrium first despite the competitive superiority of the predator (fig. $5 \mathrm{C}$ ); this is because adult predators specialize on consumers and thus predators cannot exist in their absence. The predator, however, invades soon thereafter, leading to coexistence as the only equilibrium state (fig. 5C). Cannibalism has no effect on qualitative dynamics in this scenario but does stabilize oscillations (fig. $5 C$; note Hopf bifurcation).

In the diet broadening scenario, the predator-resource equilibrium occurs as the only equilibrium state when predators are superior in resource competition in the absence of cannibalism. Interestingly, in contrast to all other scenarios, high cannibalism actually allows consumer invasion into this equilibrium at high resource productivity (i.e., threespecies coexistence; fig. 5D). Again, this is due to the previously described shift in predator stage distribution due to cannibalism; cannibalism reduces adult biomass and increases juvenile biomass, which in turn permits consumer invasion because of a reduction in top-down control (fig. A3). Thus, in total, when the predator is the superior resource competitor, cannibalism either has no effect on qualitative equilibria (complete diet shift; fig. $5 C$ ) or promotes coexistence (diet broadening; fig. 5D).

\section{Discussion}

A hallmark of structured predator-prey systems is that different predator sizes or stages often engage in different types of species interactions (Werner and Gilliam 1984; Rudolf and Lafferty 2011). Thus, influences on predator population 


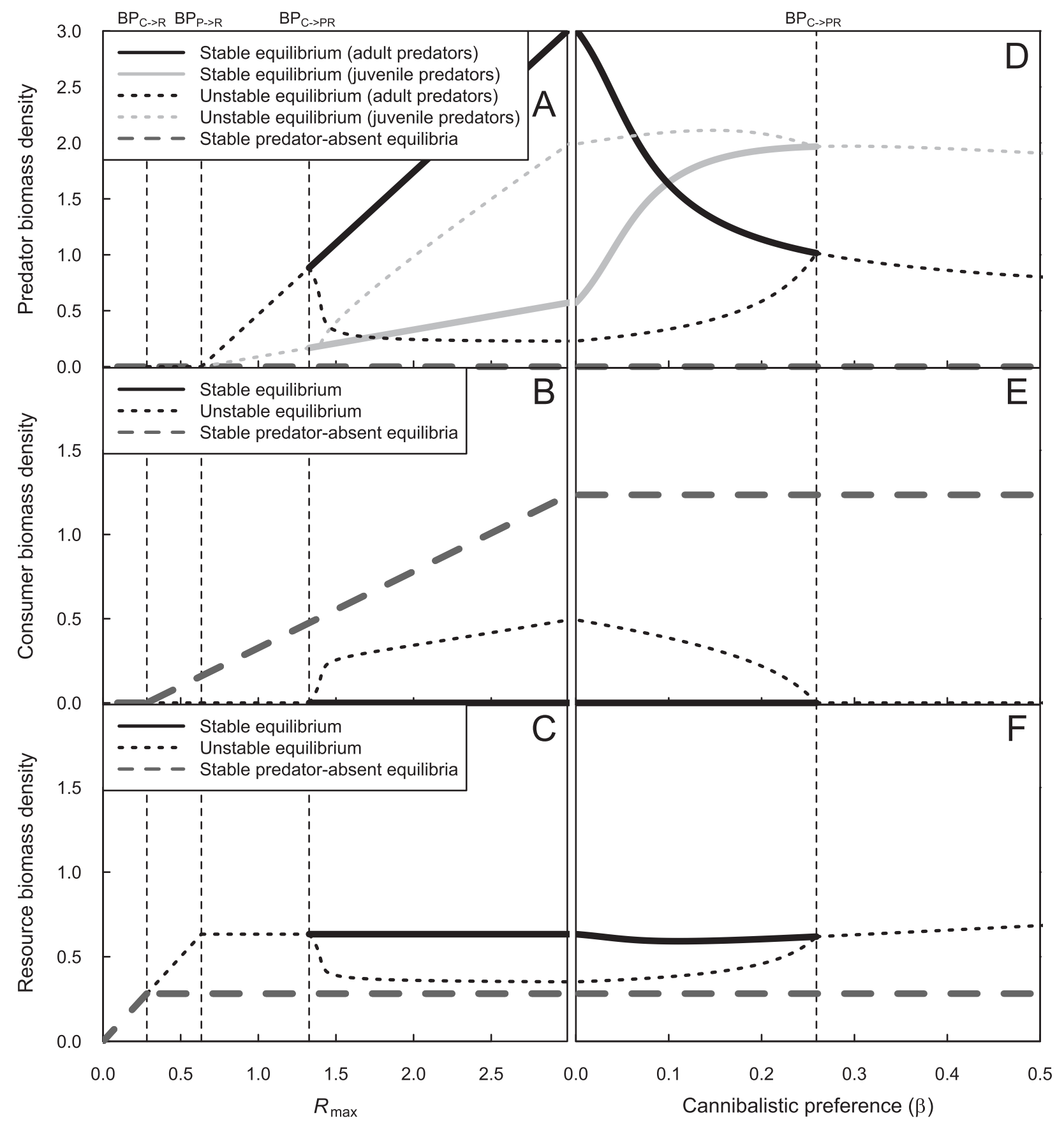

Figure 4: Equilibrium biomass as a function of resource productivity $\left(R_{\max }\right)$ when the predator undergoes ontogenetic diet broadening $(A-C)$ and effects of cannibalistic preference $(\beta)$ on these equilibria $\left(D-F\right.$; calculated at $\left.R_{\max }=3\right)$. The consumer is competitively dominant in resource consumption. Lines are as in figure 2 . In $A-C$, the consumer invasion (branching) point, $\mathrm{BP}_{\mathrm{C} \rightarrow \mathrm{R}}$, marks the productivity threshold at which consumers can invade the resource-only equilibrium, while $\mathrm{BP}_{\mathrm{P} \rightarrow \mathrm{R}}$, the predator invasion (branching) point, marks the productivity threshold at which predators can invade the resource-only equilibrium. A second branching point of the consumer, $\mathrm{BP}_{\mathrm{C} \rightarrow \mathrm{PR}}$, marks the productivity threshold at which a stable predator-resource equilibrium becomes possible (as a function of $R_{\max }$ in $A-C$ ) or disappears (as a function of $\beta$ in $D-F$ ). Stability of this predator-resource equilibrium branch changes as a result of the unstable three-species coexistence equilibrium that originates at $\mathrm{BP}_{\mathrm{C} \rightarrow \mathrm{PR}}$. Parameter settings are as in figure 3, except $\Phi=0.5$. A color version of this figure is available online. 


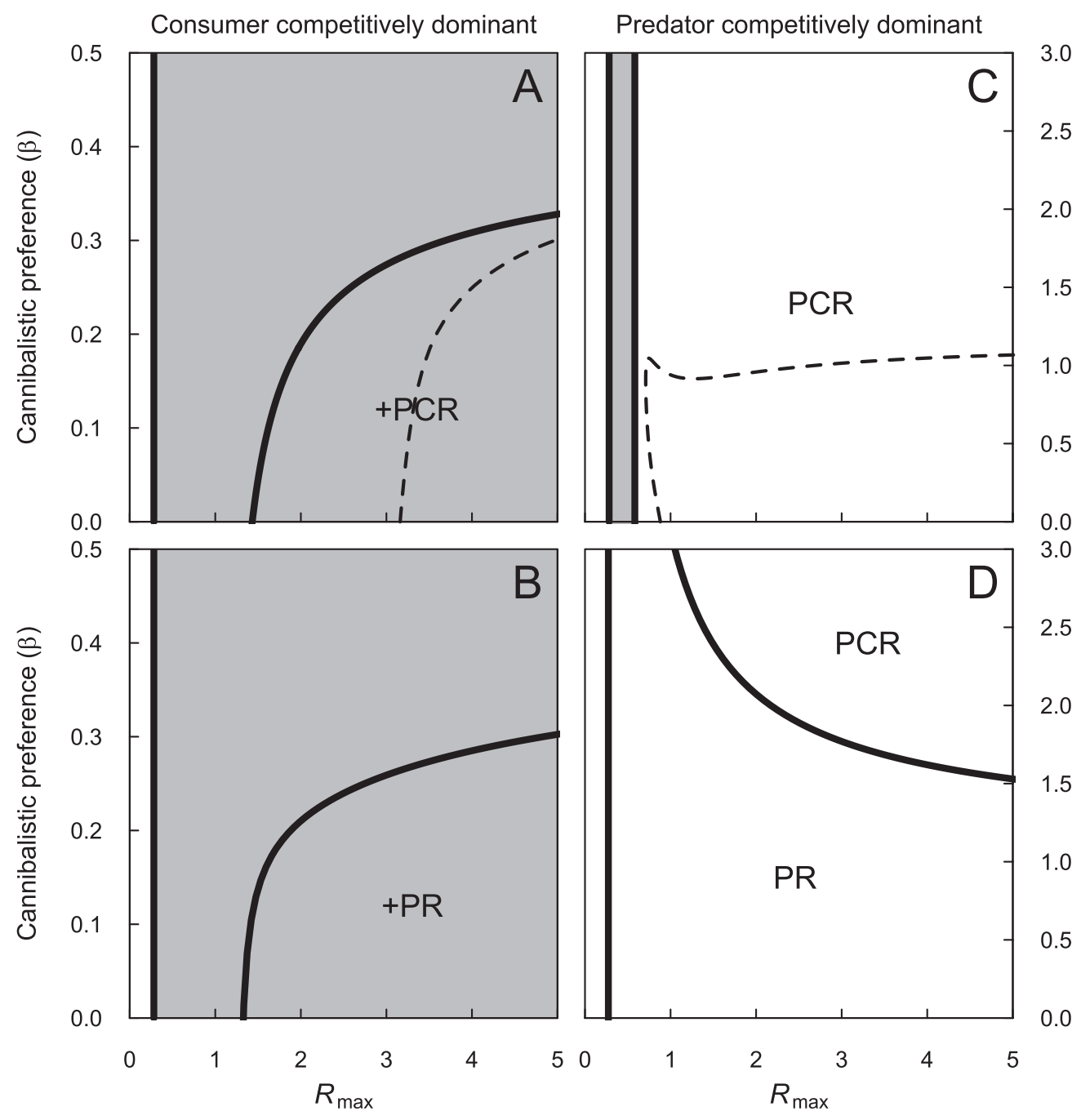

Figure 5: Equilibria dependent on resource productivity $\left(R_{\max }\right)$ and cannibalistic preference $(\beta)$ within four life-history intraguild predation scenarios: $A$, complete diet shift in predator $(\Phi=1)$, consumer as superior resource competitor $\left(m_{\mathrm{p}}=2.5\right)$; $B$, diet broadening in predator $(\Phi=0.5)$, consumer as superior resource competitor $\left(m_{\mathrm{p}}=2.5\right)$; $C$, complete diet shift in predator $(\Phi=1)$, predator as superior resource competitor $\left(m_{\mathrm{p}}=5\right)$; $D$, diet broadening in predator $(\Phi=0.5)$, predator as superior resource competitor $\left(m_{\mathrm{p}}=5\right)$. Other parameter settings are as in figure 3. Solid lines indicate shifts between different qualitative equilibria. The narrow white region on the left-hand side of all four panels represents the resource-only equilibrium. Gray shading indicates the presence of a consumer-resource (CR) equilibrium. Letters indicate the presence of different predator-present equilibria: predator-resource (PR) or predator-consumer-resource (PCR). A plus sign preceding letters indicates that these predator-present equilibria occur as alternatives to a CR equilibrium. The dashed lines in $A$ and $C$ mark the transition from a stable equilibrium point to a stable limit cycle (Hopf bifurcation), with the limit cycle occurring at higher resource productivity.

stage distribution have the capacity to shift the balance of interactions that shape long-term system dynamics. We present the novel finding that predators can short-circuit their own persistence in LHIGP systems through cannibalism, a nearly ubiquitous interaction in structured predator-prey systems (Rudolf 2007; Byström et al. 2013; Toscano et al. 2016). This occurs via a cannibalism-induced shift in the predator stage distribution from adult to juvenile biomass dominance. The loss of adult predators, solely responsible for regulating consumers, frees competitively dominant consumers from top-down control, ultimately leading to pred- ator exclusion. Predator extinction via cannibalism is possible only because of the feedback loop between predators and competitors; in a single-species system, cannibalism, a negatively density-dependent process, cannot drive extinction. We further show that this effect hinges upon the competitive superiority of consumers; when predators are competitively superior, cannibalism either has no effect on coexistence (in the complete diet shift scenario) or actually drives coexistence (in diet broadening). Thus, while previous work emphasizes the (positive) effects of cannibalism in promoting coexistence (Rudolf 2007; Ohlberger et al. 2012), our study 
demonstrates a diversity of effects of cannibalism on longterm LHIGP dynamics, depending on system properties.

\section{Ontogenetic Asymmetry in LHIGP Systems}

We show that while the community-level effects of cannibalism can be diverse, they are all driven by a single mechanism: cannibalism (and specifically juvenile predator mortality) switches the predator stage distribution from adult to juvenile biomass dominance. The loss of adult predators (and thus top-down control) either eliminates, destabilizes, or allows consumer invasion into predator-present equilibria. Such asymmetries in biomass distribution arise when the key life-history transitions, development (i.e., maturation) and reproduction, differ in their rates of biomass transfer, a phenomenon termed ontogenetic asymmetry (de Roos et al. 2007; de Roos and Persson 2013; Persson and de Roos 2013). Predator populations in LHIGP systems are dominated by adult biomass at equilibrium because of the relatively greater energy intake rate of juveniles compared to adults. Thus, maturation occurs at a greater rate than reproduction (i.e., reproduction regulation). Our analyses indicate that cannibalism actually enhances the rate of reproduction relative to maturation by increasing juvenile mortality (i.e., a positive effect of mortality; Schröder et al. 2014), reversing the ontogenetic distribution of biomass. Previous analyses support this finding, that mortality can alter population stage distribution independent of which life stage is subjected to increased mortality (de Roos et al. 2007). Such ontogenetic asymmetry is an underappreciated characteristic of structured populations that is likely widespread in nature (de Roos and Persson 2013; Persson and de Roos 2013; Reichstein et al. 2015). Our work thus provides a striking example of the importance of ontogenetic asymmetry for longterm system dynamics and further suggests that natural LHIGP systems could be more sensitive to changes in predator stage distribution than previously recognized, whether because of cannibalism or other means (e.g., human harvesting; Walters and Kitchell 2001). Specifically, any factor that increases juvenile-specific mortality (e.g., disease or a sizedependent predator) could elicit similar effects in LHIGP systems without cannibalism.

\section{Cannibalistic Preference and Coexistence}

We found that the degree of cannibalism necessary to inhibit predator persistence in LHIGP is surprisingly low: the loss of predator-present equilibria occurs at a $\sim 3: 1$ preference for heterospecific versus conspecific prey in both complete diet shift and diet broadening scenarios. While this precise cannibalistic preference is dependent on our model parameterization, the important finding is that adults can short-circuit their own persistence while still exhibiting preference for heterospecific prey. Furthermore, our parameterization is based on power-law scaling of physiological rates with body size and is thus justifiable on the basis of metabolic theory (see Hin et al. 2011 for further explanation). Models based on biomass density, such as our formulation, allow for a much more realistic parameterization than typical consumer-resource models based on individual density (Yodzis and Innes 1992). Thus, our results are certainly within the realm of biological possibility.

We can use empirical estimates of cannibalistic preference to speculate on whether predator taxa would inhibit their own persistence in LHIGP systems. Our literature review shows that cannibalistic preference varies considerably among a wide range of invertebrate and vertebrate predator taxa and can rival or even exceed interspecific predation rates (Gerber and Echternacht 2000; Yasuda et al. 2001; Burgio et al. 2005; Rudolf 2008). Byström et al. (2013), for example, found that freshwater fish often exhibit a strong preference for conspecific relative to heterospecific prey. Interestingly, this same study found that fish predators are typically competitively inferior to their prey (intermediate fish consumers; Byström et al. 2013). Our results suggest that in these fish systems, there is a high likelihood of predator exclusion from LHIGP interactions. Thus, it appears that the conditions for predator exclusion from LHIGP interactions are met in a number of natural LHIGP systems.

\section{Reconciling Theory with Nature}

Given these empirical patterns of cannibalism, our work thus poses an apparent conundrum: how can LHIGP systems with cannibalism be common in nature (Rudolf 2007; Byström et al. 2013)? The sensitivity of LHIGP to cannibalism is due in part to consumer competitive superiority: any factor that frees consumers from top-down control leads to a juvenile competitive bottleneck that inhibits predator persistence. This assumption (consumers superior to predators in resource competition) is likely upheld in many natural systems because of an ontogenetic trade-off associated with continuous growth (Werner and Gilliam 1984; Hin et al. 2011; Byström et al. 2013): continuously growing predators retain the same basic morphology while switching among resource types, and thus juvenile predators may be "burdened" by adult predatory morphology (Werner and Gilliam 1984). In contrast, specialist consumers feed solely on the basal resource over ontogeny and are thus not faced with such a trade-off.

This condition, however, is not necessarily true for species that undergo complete metamorphosis (Werner and Gilliam 1984; but see Toscano et al. 2016). Metamorphosis allows predators to completely "rebuild" their morphology over ontogeny, allowing them to potentially circumvent the same phenotypic trade-off. Thus, as an alternative scenario, we present the effects of cannibalism on LHIGP dynamics 
when predators are competitively superior to consumers in resource competition, showing that the juvenile competitive bottleneck and concomitant loss of predator-present equilibria are no longer possible. Instead, a predator-present equilibrium is the sole equilibrium state in both complete diet shift and diet broadening scenarios. In the complete diet shift scenario, this predator-present state occurs as full (three-species) coexistence, while in the diet broadening scenario, this occurs as a predator-resource equilibrium. Nevertheless, strong cannibalism in the diet broadening scenario actually allows consumer invasion into this predator-resource equilibrium, thus promoting coexistence. Rudolf (2007) demonstrates this same effect of cannibalism in a diet broadening LHIGP model with a fixed (i.e., non-food dependent) maturation rate, showing that this result is robust to model formulation. Thus, when the predator is superior in resource competition, coexistence is a likely outcome of LHIGP with cannibalism. More broadly, consumer versus predator competitive superiority is critical to predicting the long-term outcome of LHIGP interactions and could depend in part on the details of predator life-history (e.g., continuous growth vs. metamorphosis).

Other mechanisms could also allow for predator persistence in cannibalistic LHIGP systems when consumers are superior in resource competition, although these require an increase in system complexity beyond the basic LHIGP module. An extraneous (i.e., not predator-driven) increase in consumer mortality, for example, permits predator persistence in both complete diet shift and diet broadening LHIGP scenarios, despite strong cannibalism (B. J. Toscano, unpublished results). In this scenario, increased mortality reduces consumer population growth (i.e., competitive ability) and thus the ability of consumers to competitively exclude predators. Thus, it is possible that natural LHIGP interactions nested within complex food webs are more complicated than suggested by current model formulations, and this additional complexity begets the apparent stability of these interactions in nature.

Empirical ecologists may begin to test the theory we present by using multigenerational community-scale experiments. Specifically, to test the effects of cannibalism on LHIGP system dynamics, we suggest using congeneric predators that differ in their degree of cannibalism (e.g., copepods; Toscano et al. 2016) nested in otherwise identical LHIGP systems. While different predator species may vary in their precise life-history parameters or feeding rates, our theory is robust to these differences, predicting qualitatively different dynamics in the absence and presence of cannibalism. Specifically, predator persistence should not be possible in the presence of a cannibalistic predator, whereas in the absence of cannibalism, predator-present community states are predicted. Juvenile mortality is the primary process behind the collapse of predator-present stable states, and thus the experimental harvest of juveniles should elicit the same community response as cannibalism while corroborating the stagedependent mechanism.

Cannibalism is predicted to result in predator-absent LHIGP states, and so the effects of cannibalism could be more difficult to test within intact natural communities. Nevertheless, additional analyses suggest that in LHIGP systems with low cannibalism, increasing consumer mortality (e.g., via a removal manipulation) should shift the predator to a more adult-dominated stage distribution (i.e., resembling the dynamics of an LHIGP system without cannibalism). Furthermore, consumers should exhibit complete biomass compensation in response to increased mortality. Such community responses to increased consumer mortality would identify cannibalism as a key regulatory interaction. We emphasize, however, that long-term multigenerational studies of LHIGP dynamics are rare, and any such study would significantly advance our understanding of stage-structured community dynamics.

\section{Conclusions}

This work demonstrates the potential for strong effects of cannibalism on the long-term outcomes of stage-structured species interactions, adding to just a handful of studies that have investigated the effects of cannibalism on complex communities (Wahlström et al. 2000; Persson et al. 2003; Rudolf 2007; Ohlberger et al. 2012). Such a paucity of research attention is surprising, given that cannibalism is nearly ubiquitous in structured populations (Fox 1975; Polis 1981; Polis and Myers 1985). Claessen et al. (2004, p. 339) suggest that this lack of study is because "the complexity of (structured) cannibalistic-population models makes extensions to multispecies systems a daunting task." Nevertheless, we believe that this is a task worth taking on, in order to better understand how communities might respond to the full range of biotic interactions in nature.

\section{Acknowledgments}

We wish to thank A. de Roos for helpful feedback that improved this work. This work was supported by an Arnold O. Beckman Postdoctoral Fellowship to B.J.T. and National Science Foundation grant DEB-1256860 to V.H.W.R. V.H. was supported by funding from the European Research Council (ERC) under the European Union's Seventh Framework Programme (FP/2007-2013)/ERC grant agreement no. 594 322814.

\section{Literature Cited}

Abrams, P. A. 2011. Simple life-history omnivory: responses to enrichment and harvesting in systems with intraguild predation. American Naturalist 178:305-319. 
Baras, E., F. Tissier, J.-C. Philippart, and C. Mélard. 1999. Sibling cannibalism among juvenile vundu under controlled conditions. II. Effect of body weight and environmental variables on the periodicity and intensity of type II cannibalism. Lournal of Fish Biology 54: 106-118.

Brose, U., T. Jonsson, E. L. Berlow, P. Warren, C. Banasek-Richter, L. F. Bersier, J. L. Blanchard, et al. 2006. Consumer-resource body-size relationships in natural food webs. Ecology 87:2411-2417.

Burgio, G., F. Santi, and S. Maini. 2005. Intra-guild predation and cannibalism between Harmonia axyridis and Adalia bipunctata adults and larvae: laboratory experiments. Bulletin of Insectology 58:135140.

Byström, P., P. Ask, J. Andersson, and L. Persson. 2013. Preference for cannibalism and ontogenetic constraints in competitive ability of piscivorous top predators. PLoS ONE 8(7):e70404. doi:10.1371 /journal.pone.0070404.

Chesson, J. 1978. Measuring preference in selective predation. Ecology 59:211-215.

Claessen, D., A. M. de Roos, and L. Persson. 2000. Dwarfs and giants: cannibalism and competition in size-structured populations. American Naturalist 155:219-237.

. 2004. Population dynamic theory of size-dependent cannibalism. Proceedings of the Roval Society B 271:333-340.

de Roos, A. M., and L. Persson. 2013. Population and community ecology of ontogenetic development. Princeton University Press, Princeton, NJ.

de Roos, A. M., T. Schellekens, T. van Kooten, K. van de Wolfshaar, D. Claessen, and L. Persson. 2007. Food-dependent growth leads to overcompensation in stage-specific biomass when mortality increases: the influence of maturation versus reproduction regulation. American Naturalist 170:E59-E76.

2008. Simplifying a physiologically structured population model to a stage-structured biomass model. Theoretical Population Biology 73:47-62.

Dhooge, A., W. Govaerts, and Y. A. Kuznetsov. 2003. MATCONT: a MATLAB package for numerical bifurcation analysis of ODEs. ACM Transactions on Mathematical Software 29:141-164.

Dörner, H., S. Hülsmann, F. Hölker, C. Skov, and A. Wagner. 2007. Size-dependent predator-prey relationships between pikeperch and their prey fish. Ecology of Freshwater Fish 16:307-314.

Duelli, P. 1981. Is larval cannibalism in lacewings adaptive? (Neuroptera: Chrysopidae). Researches on Population Ecology 23:193209.

Fox, L. R. 1975. Cannibalism in natural populations. Annual Review of Ecology and Systematics 6:87-106.

Gagné, I., D. Coderre, and Y. Mauffette. 2002. Egg cannibalism by Coleomegilla maculata lengi neonates: preference even in the presence of essential prey. Ecological Entomology 27:285-291.

Gerber, G. P., and A. C. Echternacht. 2000. Evidence for asymmetrical intraguild predation between native and introduced Anolis lizards. Oecologia 124:599-607.

Hin, V., T. Schellekens, L. Persson, and A. M. de Roos. 2011. Coexistence of predator and prey in intraguild predation systems with ontogenetic niche shifts. American Naturalist 178:701-714.

Leonardsson, K. 1991. Effects of cannibalism and alternative prey on population dynamics of Saduria entomon (Isopoda). Ecology 72: 1273-1285.

Macpherson, E., and A. Gordoa. 1994. Effect of prey densities on cannibalism in Cape hake (Merluccius capensis) off Namibia. Marine Biology 119:145-149.
MacRae, I., and B. Croft. 1997. Intra-and interspecific predation by adult female Metaseiulus occidentalis and Typhlodromus pyri (Acari: Phytoseiidae) when provisioned with varying densities and ratios of Tetranychus urticae (Acari: Tetranychidae) and phytoseiid larvae. Experimental and Applied Acarology 21:235-246.

Mahe, K., R. Amara, T. Bryckaert, M. Kacher, and J.-M. Brylinski. 2007. Ontogenetic and spatial variation in the diet of hake (Merluccius merluccius) in the Bay of Biscay and the Celtic Sea. ICES Journal of Marine Science 64:1210-1219.

Miller, T. E., and V. H. Rudolf. 2011. Thinking inside the box: community-level consequences of stage-structured populations. Trends in Ecology and Evolution 26:457-466.

Ohlberger, J., Ø. Langangen, N. C. Stenseth, and L. A. Vøllestad. 2012. Community-level consequences of cannibalism. American Naturalist 180:791-801.

Oksanen, L., S. D. Fretwell, J. Arruda, and P. Niemela. 1981. Exploitation ecosystems in gradients of primary productivity. American Naturalist 118:240-261.

Orr, B. K., W. W. Murdoch, and J. R. Bence. 1990. Population regulation, convergence, and cannibalism in Notonecta (Hemiptera). Ecology 71:68-82.

Park, S. R., J. Y. Jeong, and D. Park. 2005. Cannibalism in the Korean salamander (Hynobius leechii: Hynobiidae, Caudata, Amphibia) larvae. Integrative Biosciences 9:13-18.

Persson, L., and A. M. de Roos. 2013. Symmetry breaking in ecological systems through different energy efficiencies of juveniles and adults. Ecology 94:1487-1498.

Persson, L., A. M. de Roos, D. Claessen, P. Byström, J. Lövgren, S. Sjögren, R. Svanbäck, E. Wahlström, and E. Westman. 2003. Gigantic cannibals driving a whole-lake trophic cascade. Proceedings of the National Academv of Sciences of the USA 100:4035-4039.

Peters, R. H. 1983. The ecological implications of body size. Cambridge University Press, Cambridge.

Pimm, S. L., and J. C. Rice. 1987. The dynamics of multispecies, multi-life-stage models of aquatic food webs. Theoretical Population Biology 32:303-325.

Polis, G. A. 1981. The evolution and dynamics of intraspecific predation. Annual Review of Ecology and Systematics 12:225-251.

Polis, G. A., and C. A. Myers. 1985. A survey of intraspecific predation among reptiles and amphibians. Journal of Herpetology 19:99-107.

Reichstein, B., L. Persson, and A. M. de Roos. 2015. Ontogenetic asymmetry modulates population biomass production and response to harvest. Nature Communications 6:6441. doi:10.1038/ncomms7441. Rudolf, V. H. W. 2007. The interaction of cannibalism and omnivory: consequences for community dynamics. Ecology 88:2697-2705.

2008. Impact of cannibalism on predator-prey dynamics: sizestructured interactions and apparent mutualism. Ecology 89:16501660.

Rudolf, V. H. W., and K. D. Lafferty. 2011. Stage structure alters how complexity affects stability of ecological networks. Ecology Letters 14:75-79.

Rudolf, V. H. W., N. L. Rasmussen, C. J. Dibble, and B. G. Van Allen. 2014. Resolving the roles of body size and species identity in driving functional diversity. Proceedings of the Roval Society B 281: 20133203. doi:10.1098/rspb.2013.3203.

Santi, F., G. Burgio, and S. Maini. 2003. Intra-guild predation and cannibalism of Harmonia axyridis and Adalia bipunctata in choice conditions. Bulletin of Insectology 56:207-210.

Schausberger, P. 1999. Predation preference of Typhlodromus pyri and Kampimodromus aberrans (Acari: Phytoseiidae) when offered 
con- and heterospecific immature life stages. Experimental and Applied Acarology 23:389-398.

Schausberger, P., and B. A. Croft. 2000. Cannibalism and intraguild predation among phytoseiid mites: are aggressiveness and prey preference related to diet specialization? Experimental and Applied Acarology 24:709-725.

Schröder, A., A. van Leeuwen, and T. C. Cameron. 2014. When less is more: positive population-level effects of mortality. Trends in Ecology and Evolution 29:614-624.

Streams, F. A. 1992. Intrageneric predation by Notonecta (Hemiptera: Notonectidae) in the laboratory and in nature. Annals of the Entomological Society of America 85:265-273.

Toscano, B. J., V. Hin, and V. H. W. Rudolf. 2017. Data from: Cannibalism and intraguild predation community dynamics: coexistence, competitive exclusion, and the loss of alternative stable states. American Naturalist, Dryad Digital Repository, http://dx.doi.org/10.5061 /dryad.vj12j.

Toscano, B. J., B. R. Rombado, and V. H. W. Rudolf. 2016. Deadly competition and life-saving predation: the potential for alternative stable states in a stage-structured predator-prey system. Proceedings of the Roval Society B 283:20161546. doi:10.1098/rspb.2016 .1546 .

van de Wolfshaar, K. E., A. M. de Roos, and L. Persson. 2006. Sizedependent interactions inhibit coexistence in intraguild predation systems with life-history omnivory. American Naturalist 168:62-75.
Wahlström, E., L. Persson, S. Diehl, and P. Byström. 2000. Sizedependent foraging efficiency, cannibalism and zooplankton community structure. Oecologia 123:138-148.

Walters, C., and J. F. Kitchell. 2001. Cultivation/depensation effects on juvenile survival and recruitment: implications for the theory of fishing. Canadian Journal of Fisheries and Aquatic Sciences 58:39-50.

Walzer, A., and P. Schausberger. 1999. Cannibalism and interspecific predation in the phytoseiid mites Phytoseiulus persimilis and Neoseiulus californicus: predation rates and effects on reproduction and juvenile development. Biocontrol 43:457-468.

Werner, E. E. 1988. Size, scaling, and the evolution of complex life cycles. Pages 60-81 in B. Ebenman and L. Persson, eds. Size-structured populations: ecology and evolution. Springer, Berlin.

Werner, E. E., and J. F. Gilliam. 1984. The ontogenetic niche and species interactions in size-structured populations. Annual Review of Ecology and Systematics 15:393-425.

Yasuda, H., T. Kikuchi, P. Kindlmann, and S. Sato. 2001. Relationships between attack and escape rates, cannibalism, and intraguild predation in larvae of two predatory ladybirds. Journal of Insect Behavior 14:373-384.

Yodzis, P., and S. Innes. 1992. Body size and consumer-resource dynamics. American Naturalist 139:1151-1175.

Associate Editor: Benjamin M. Bolker Editor: Alice A. Winn

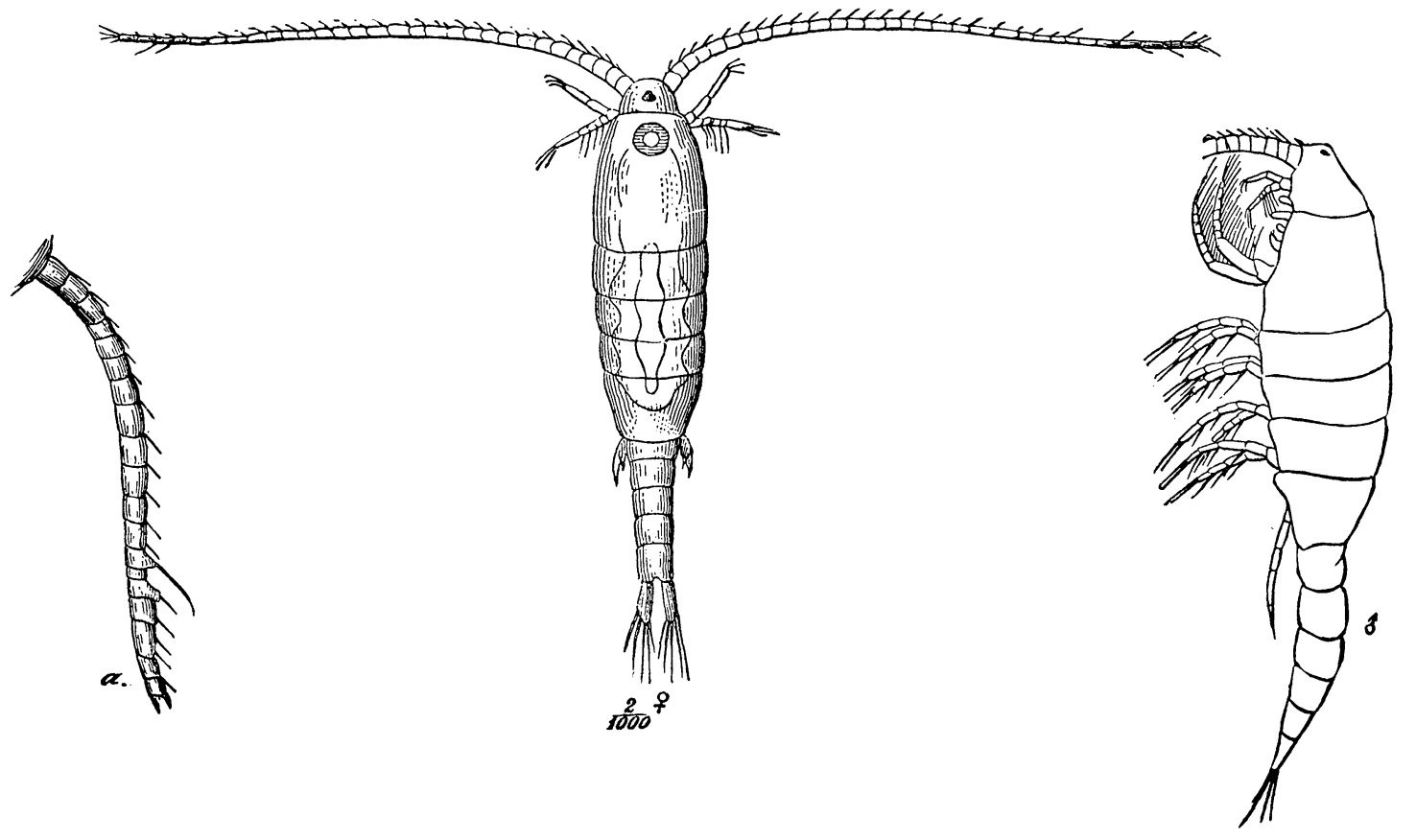

\footnotetext{
"The collector of fresh-water specimens is constantly meeting unexpected forms, especially among the smaller organisms, and of these no order of animals furnishes a wider variety or more curious adaptations than the fresh-water Crustaceans embraced in the old group Entomostraca. . .." Figured: "Diaptomus longicornis Herrick, back view of female and side view of male; $a$, basal portion of male antennæ showing enlarged segments preceding geniculating joint." From “Fresh-Water Entomostraca” by C. L. Herrick (The American Naturalist, 1879, 13:620-628).
} 